\title{
Iterative Reconstruction of the Magnetization and Charge Density using Vector Field Electron Tomography
}

\author{
K. Aditya Mohan ${ }^{1}$, Prabhat K. C. ${ }^{2}$, Charudatta Phatak $^{3}$, Marc De Graef ${ }^{2}$, Charles A. Bouman ${ }^{1}$ \\ ${ }^{1}$ Dept. of Electrical and Computer Engineering, Purdue Univ., West Lafayette IN 47907, USA \\ ${ }^{2}$ Dept. of Materials Science and Engineering, Carnegie Mellon Univ., Pittsburgh PA 15213, USA \\ ${ }^{3}$ Argonne National Laboratory, Lemont IL 60439, USA
}

Vector field electron tomography (VFET) is used to reconstruct the 3D spatial distribution of the magnetic vector potential and electrostatic potential around an object [1]. The magnetic and electrostatic potentials induce phase changes in the incoming electron wave such that the phase change is linearly related to the radon transform of the two potentials. The phase image at each tilt angle is then recovered from detector measurements at multiple defocus values [1] and the object is reconstructed by tomographic inversion of the retrieved phase images. To uniquely reconstruct both the magnetic and electrostatic potentials, it is necessary to acquire tilt series data over the full $360^{\circ}$ range [1]. Furthermore, to uniquely recover the three components of the magnetic vector potential it is necessary to acquire tilt series data at two orthogonal rotation axes [1].

Traditionally, the reconstruction is done by direct analytical inversion of the magnetic and electrostatic components of the phase images [1-3]. Such analytical methods are sensitive to noise and result in reconstruction artifacts due to the limited angular range of the tilt series measurements [1]. Furthermore, this approach does not recover the distribution of the magnetization (total dipole moment per unit volume) and charge density in the object that is responsible for the magnetic and electrostatic potentials.

In this paper, we present a model-based iterative reconstruction algorithm (MBIR) that directly reconstructs the magnetization and charge density from the recovered phase data. The proposed algorithm is based on the minimization of a cost function consisting of a forward model term and a prior model term that enforces sparsity in the reconstructed values. The forward model models the physics of phase change as a function of the magnetization and charge density in the object. In comparison to analytical approaches, MBIR algorithms have been shown to be robust to noise, limited angle, and sparsely sampled data [4].

To derive the reconstruction algorithm, it is first necessary to express the phase change, $\phi(r)$ at position vector $r$, as a function of the magnetization, $M(r)$, and charge density, $\rho(r)$, of the object. As the electron wave propagates through space, it undergoes a phase shift under the influence of the magnetic vector potential, $A(r)$, and electrostatic potential, $V(r)$, as,

$$
\phi\left(r_{\perp}\right)=-\frac{e}{\hbar} \int A\left(r_{\perp}+l w\right) \cdot w d l+\frac{\pi}{\lambda E} \int V\left(r_{\perp}+l w\right) d l
$$

where $w$ is the unit vector in the direction of propagation, $r_{\perp}$ is the position vector in the detector plane, $\lambda$ is the wavelength of the electron wave, $E$ is the relativistic electron accelerating potential, $\hbar$ and $e$ are constants [1]. The magnetic vector potential, $A(r)$, and the electrostatic potential, $V(r)$, are related to the magnetization, $M(r)$, and the charge density, $\rho(r)$ as [5],

$$
A(r)=\frac{\mu_{0}}{4 \pi} \iiint d r^{\prime} M\left(r^{\prime}\right) \times \frac{r-r^{\prime}}{\left|r-r^{\prime}\right|^{3}}, \text { and } V(r)=\frac{1}{4 \pi \epsilon_{0}} \iiint \frac{\rho\left(r^{\prime}\right)}{\left|r-r^{\prime}\right|} d r^{\prime},
$$


where $\times$ denotes the cross-product between two vectors, $\mu_{0}$ is the permeability of vacuum, $\epsilon_{0}$ is the permittivity of free space, $r$ and $r^{\prime}$ are the position vectors in 3D space.

Let $\mathbf{x}$ be the vector that contains the voxel values of the charge density and the three components of the magnetization of the discretized object. Similarly, let $\mathbf{z}$ be the vector that contains the voxel values of the electrostatic potential and the three components of the magnetic vector potential in and around the object. Then, we can write $\mathbf{z}=H \mathbf{x}$ where $H$ is a matrix that implements the convolution integrals in (2). Let $\mathbf{y}_{i}$ be the vector of phase shifts of the electron wave at the $i^{t h}$ tilt angle. Then, the relation between the potentials $\mathbf{z}$ and the phase shifts $\mathbf{y}_{i}$ is given by $\mathbf{y}_{i}=A_{i} \mathbf{z}+\mathbf{n}_{i}$ where $A_{i}$ is the projection matrix that implements the line integrals in (1) and $\mathbf{n}_{i}$ is the noise vector. Thus, the relation between $\mathbf{y}_{i}$ and $\mathbf{x}$ is given by $\mathbf{y}_{i}=A_{i} H \mathbf{x}+\mathbf{n}_{i}$.

The reconstruction is then obtained by minimizing the sum of squared errors between the phase shifts, $\mathbf{y}_{i}$, and its estimates, $A_{i} H \mathbf{x}$, and a regularizing prior function, $R(\mathbf{x})$, as shown below -

$$
\hat{\mathbf{x}}=\operatorname{argmin}_{\mathbf{x}}\left\{\sum_{i=1}^{M}\left\|\mathbf{y}_{i}-A_{i} H \mathbf{x}\right\|^{2}+R(\mathbf{x})\right\}
$$

where $M$ is the total number of tilt angles for all the rotation axes. The above form of the cost function is computationally intensive to minimize directly. So, we use variable splitting and formulate (3) as a constrained optimization problem of the form $\hat{\mathbf{x}}=\operatorname{argmin}_{\mathbf{x}, \mathbf{z}}\left\{\sum_{i=1}^{M}\left\|\mathbf{y}_{i}-A_{i} \mathbf{z}\right\|^{2}+R(\mathbf{x})\right\}$ such that $\mathbf{z}=H \mathbf{x}$. To solve the above constrained form of the optimization problem, we use the theory of alternate direction method of multipliers (ADMM) [6] to express the original problem as an iterative solution of two simpler optimization problems as shown in Fig. 1. In Fig. 1, the data y consists of the recovered phase shifts at all the tilt angles and rotation axes. The tomographic inversion step is solved by a simple modification of the algorithm presented in [4]. The deconvolution step is solved directly using gradient based methods. The two optimization steps are performed in an iterative manner until the algorithm converges. Example vector field reconstructions will be provided.

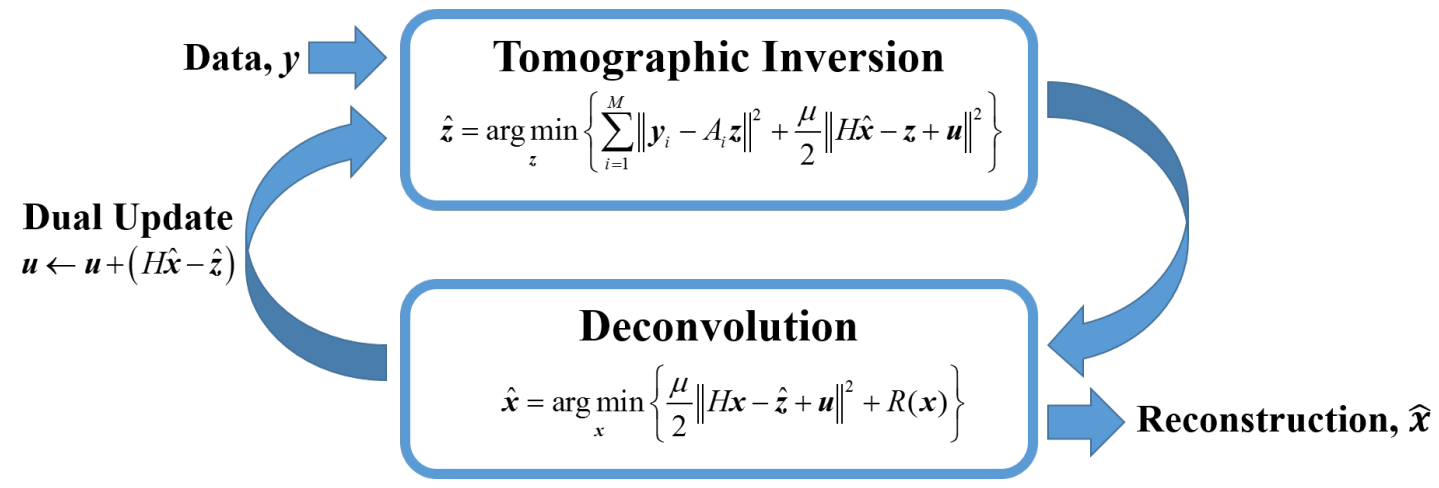

Figure 1: Reconstruction of the magnetization and charge density by solving the optimization problem in (3).

\section{References}

[1] C. Phatak et al, Ultramicroscopy 108-6 (2008), p. 503-513.

[2] V. Stolojan et al, Inst. Phys. Conf. Ser. 168 (2001), p. 243.

[3] S. J. Lade et al, Opt. Commun. 253 (2005), p. 392.

[4] K. A. Mohan et al, IEEE Tran. on Comp. Imaging 1-2 (2015), p. 96111.

[5] J. D. Jackson in "Classical Electrodynamics", ed. 3, (Wiley).

[6] S. Boyd et al, Foundations and Trends in Machine Learning 3-1 (2011), p. 1122.

[7] Research supported by the Air Force Office of Scientific Research, MURI contract \# FA9550-12-1-0458. 